\title{
Avaliação da qualidade da assistência de enfermagem: qual a melhor estratégia para o repasse dos resultados?
}

\section{Evaluation of nursing assistance quality: which is the best strategy for passing the results on?}

\author{
Silvia Regina Mattias ${ }^{1}$; Marli Terezinha Oliveira Vannuchi²; \\ Dagmar Willamowis Vituri³; José Carlos Dalmas ${ }^{4}$
}

\begin{abstract}
Resumo
O controle de qualidade na assistência de enfermagem envolve o estabelecimento de padrões baseados em um modelo de cuidado que deve subsidiar o desenvolvimento de medidas passíveis de serem alcançadas, e sirvam como guia das ações aos profissionais. O objetivo deste trabalho foi determinar a opinião da equipe de enfermagem de uma unidade de internação no Hospital Universitário Regional do Norte Paraná, Londrina, quanto à forma utilizada pela Assessoria de Controle de Qualidade de Assistência de Enfermagem (ACQAE), para repassar relatórios de avaliação da qualidade de assistência local e levantar propostas de adequação. Tratou-se de estudo descritivo exploratório, de abordagem quantitativa. O instrumento de coleta de dados consistiu em questionário composto por 21 perguntas, relacionadas ao processo de qualidade de assistência de enfermagem, realizada nesta unidade de internação. Os resultados mostraram que $81,8 \%$ consideraram os dados levantados pela ACQAE importantes para a prática profissional, $44,6 \%$ forma de repasse do resultado insuficiente, $65 \%$ sugeriram que o enfermeiro da unidade deve fazer feedback aos funcionários. O estudo confirmou a hipótese levantada anteriormente de que as equipes de enfermagem da unidade estudada, têm preferência por um profissional com vivência prática de Enfermagem para realizar o feedback, ou seja, o enfermeiro. A realização deste processo por outro profissional ou estagiário foi considerada insuficiente pelas equipes.
\end{abstract}

Palavras-chave: Qualidade de assistência à saúde. Educação em enfermagem. Equipe de enfermagem.

\begin{abstract}
Quality control in nursing assistance involves the establishment of patterns based on a model of care that must support the development of measures that can be achieved and serve as a guide of actions to professionals. The purpose of this work was to determine the opinion of a nursing staff of an admittance unit at the Regional University Hospital of Northern Paraná, Londrina, concerning the way that was used by the Quality Control Advisory Body of Nursing Assistance (ACQAE) to pass on evaluation reports of the local assistance quality and propose an adequacy. This was a descriptive-exploratoryquantitative study. The data gathering instrument was a questionnaire with 21 questions, related to the quality process of nursing assistance, carried out at this admittance unit. Results showed that $81.8 \%$ considered the data surveyed by ACQAE important for the professional practice; $44.6 \%$ considered the way the results were passed on insufficient; $65 \%$ suggested that the nurse working in this unit should give feedback to the other employees. The study confirmed the hypothesis previously considered that

\footnotetext{
1 Graduanda do $4^{\circ}$ ano do Curso de Enfermagem da Universidade Estadual de Londrina (UEL). E-mail: silmattias@yahoo.com. br

2 Prof ${ }^{\mathrm{a}}$. Dra ${ }^{\mathrm{a}}$. do Departamento de Enfermagem - UEL.

3 Profa. Ms. da Assessoria de Controle de Qualidade do HU.

4 Prof. Dr. do Departamento de Matemática Aplicada - UEL.
} 
the nursing staff of the studied unit prefers a professional with previous experience in nursing to carry out the feedback, that is, the nurse. The accomplishment of this process by another professional or intern was considered insufficient by the teams of experts.

Key words: Health care quality. Nursing education. Nursing staff.

\section{Introdução}

A preocupação com a qualidade e os métodos para controlá-la de forma sistemática, tem por objetivo assegurar resultados que atendam aos requisitos dos projetos concebidos. Essa evolução decorre do desenvolvimento da Medicina e deuse com o avanço da história, e sofreu inúmeras influências da era industrial: diante da crescente concorrência e a maior complexidade dos processos produtivos, houve cuidados com a padronização e a inspeção de produtos (NEVES; NEVES, 2000). Essa preocupação, inicialmente introduzida no setor industrial expandiu-se para o setor de prestação de serviços, incluindo o setor de saúde (BRAZ, 2002).

Em relação ao setor de saúde, o Brasil dos anos 80 viu-se pressionado frente às novas realidades sociais. As dificuldades econômicas, a evolução tecnológica e a criação de normas governamentais subsidiaram os usuários/clientes/pacientes a exercerem seus direitos de cidadãos e obter um serviço de saúde com qualidade. Com isso, algumas instituições foram levadas a repensar a maneira de administrar e adotar um gerenciamento da qualidade (HADDAD, 2004).

$\mathrm{Na}$ enfermagem, a sua precursora Florence Nightingale, já no século XIX, salientou-se pela preocupação com a melhoria da qualidade do cuidado dispensado aos soldados feridos na Guerra da Criméia, além de perceber a importância da qualidade do ambiente no qual se desenvolvia o cuidado (MALIK, 2004).

Antunes e Trevizan (2000) acrescentam que o enfermeiro, como sujeito controlador e executor dos serviços hospitalares, adota o gerenciamento da qualidade, com vistas a alcançar um padrão aceitável de assistência e, assim também, atender às expectativas dos pacientes.

Nonino (2006) acredita que a preocupação com a melhoria dos processos de produção das ações vem ganhando espaço entre os gerentes e pesquisadores de enfermagem, e acrescenta que a qualidade dos serviços de saúde prestada à população, depende da competência técnica e da habilidade de comunicação e interação da equipe com o usuário/cliente/ paciente. No mesmo sentido, Balbueno e Nozawa (2004) observam que a qualidade dos serviços de saúde está diretamente relacionada ao desempenho do pessoal de enfermagem. O acompanhamento desse desempenho promoveria desenvolvimento de recursos humanos, e garantiria a qualidade na assistência oferecida.

Com o objetivo de implementar um modelo de avaliação da qualidade do cuidado, a Diretoria de Enfermagem do Hospital Universitário Regional do Norte do Paraná (HURNP), no ano de 1998, com base em estudos de Cianciarullo (1997) e Cianciarullo, Gualda e Meleiro (1998), criou a Assessoria de Controle de Qualidade de Assistência de Enfermagem (ACQAE), com a finalidade de avaliar a assistência prestada ao cliente, monitorar os processos de trabalho e subsidiar as atividades de educação permanente. A preocupação do modelo foi direcionar as necessidades de aperfeiçoamento das equipes identificadas por meio das avaliações sistematizadas (HADDAD, 2004).

As avaliações compreendem a entrevista com o paciente internado, observação estruturada deste paciente e ambiente, mais a análise das suas prescrições de enfermagem. Este processo é desenvolvido por alunos do $3^{\circ}$ ano do Curso de Graduação em Enfermagem da Universidade Estadual de Londrina (UEL), após capacitação, 
em caráter de estágio extracurricular (HADDAD, 2004).

$\mathrm{Na}$ condição de discente de graduação em enfermagem da UEL e estagiária da ACQAE no período de março de 2006 até março de 2007, participamos efetivamente desse método de avaliação, vivenciando a avaliação da qualidade de assistência segundo os moldes da ACQAE no âmbito do processo de trabalho da equipe de enfermagem do hospital em questão.

A ACQAE conta com apenas um enfermeiro e este tem a responsabilidade de tabular os dados coletados e elaborar os relatórios com os resultados das avaliações por unidade. Compete aos estagiários retornar para as unidades avaliadas trimestralmente, e apresentar os resultados obtidos pela equipe de enfermagem, discutindo a respeito dos aspectos positivos e negativos levantados.

Após o primeiro "feedback", verificou-se que nas avaliações seguintes, a melhora na qualidade do cuidado era relativa e temporária, pois em pouco tempo as falhas voltavam a ser observadas. Esse fato sugere falta de envolvimento da equipe de enfermagem com a busca pela melhoria contínua do padrão de atendimento aos pacientes.

Diante dessa verificação, passamos a questionar se a razão desse aparente descompromisso dos servidores da enfermagem teria relação com a metodologia utilizada para o repasse dos resultados alcançados pelas equipes das unidades avaliadas. Adotou-se como hipótese se seria mais apropriado que os resultados das avaliações da ACQAE fossem repassados por profissional com vivência prática da enfermagem, pois o feedback, em relação às falhas detectadas na assistência, possivelmente, teria melhor aceitabilidade por parte dos funcionários, pois os alunos não possuem vivência do cotidiano da prática profissional.

Com base no exposto, surgiu a motivação para o desenvolvimento do presente estudo, que teve como objetivo, identificar a opinião da equipe de enfermagem da unidade de internação médicocirúrgica masculina do HURNP, em relação à forma utilizada pela ACQAE para repassar os resultados dos relatórios de avaliação da qualidade da assistência de enfermagem da unidade, bem como, levantar propostas de adequação a este método.

\section{Material e métodos}

Trata-se de um estudo descritivo exploratório, de abordagem quantitativa, desenvolvido na unidade de internação médico-cirúrgica, de um hospital universitário público do Norte do Paraná, que atende pacientes do sexo masculino acima de 12 anos de idade, das mais diversas especialidades e graus de dependência de enfermagem. A unidade é composta por 74 leitos divididos em 13 enfermarias, sendo 11 delas com seis leitos, uma com sete leitos para pacientes colonizados por microorganismos multirresistentes e um com um leito destinado a isolamento. A unidade conta com um quadro de 70 servidores de enfermagem, lotados nas escalas dos períodos matutino, vespertino e noturno.

A população de estudo incluiu todos os 70 funcionários, sujeitos que compõem as equipes de enfermagem da referida unidade de internação.

O instrumento de coleta constituiu-se de um questionário composto por 21 perguntas relacionadas ao processo de avaliação da qualidade da assistência de enfermagem, sendo, três abertas, 12 fechadas e cinco mistas. $\mathrm{O}$ instrumento foi validado por meio de pré-teste, realizado com uma enfermeira, um auxiliar de enfermagem e dois técnicos de enfermagem (Apêndice 1).

Os dados foram coletados no período de julho a setembro de 2007. O instrumento de pesquisa foi entregue em mãos, a cada funcionário, durante sua jornada de trabalho.

Os dados coletados foram tabulados em planilhas

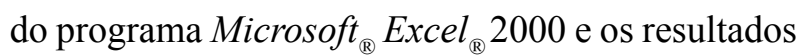
foram apresentados na forma de tabela e figuras. 
O projeto de pesquisa foi submetido à aprovação da Diretoria de Enfermagem do HURNP e à apreciação do Comitê de Ética da Universidade Estadual de Londrina, sendo aprovado mediante o Parecer $n^{\circ}$ 079/07, em conformidade com a Resolução 196/96, do Conselho Nacional de Saúde (BRASIL, 1996) (Anexo).

\section{Resultados e discussão}

Do total de 70 servidores da equipe de enfermagem da unidade em estudo, 94,3\% responderam ao instrumento de pesquisa. Dentre os que não responderam, um se negou a participar, e os demais não foram encontrados por motivo de licença.

Os dados referentes à caracterização sóciodemográfica da equipe de enfermagem da unidade em estudo estão apresentados na Tabela 1.

Tabela 1. Distribuição do número e percentual dos servidores segundo as variáveis sócio-demográficas. Londrina, PR, 2007.

\begin{tabular}{llll}
\hline Variáveis & Faixa ou tipo & Freqüência & \% \\
\hline \multirow{2}{*}{ Sexo } & Masculino & 30 & 45,5 \\
& Feminino & 36 & 54,5 \\
& Solteiro & & \\
Casado & 09 & 13,6 \\
& Viúvo & 44 & 66,7 \\
& Divorciado/separado & 09 & 13,6 \\
& Outros & 03 & 4,6 \\
& & 01 & 1,5 \\
Faixa Etária & Até 20 anos & 00 & \\
& 21 a 50 anos & 54 & 82,0 \\
& 51 anos ou mais & 12 & 18,0 \\
& & & \\
& Nenhum & 07 & 10,5 \\
Número de filhos & 1 filho 2 a 3 filhos & 18 & 27,0 \\
& acima de 3 filhos & 40 & 61,0 \\
& & 01 & 1,5 \\
\hline
\end{tabular}

O perfil dos participantes da pesquisa aponta que a maioria é do sexo feminino, casada, com uma média de dois a três filhos, mostrando que além das atividades profissionais, possuem outras atribuições relacionadas à maternidade. A média da faixa etária encontrada foi de 42,6 anos, o que pode indicar maior conhecimento e experiência profissional. Em relação ao tempo de atuação na área encontrou-se uma média de 11,8 anos de atuação, vinculados à instituição.

Quanto à categoria profissional, a equipe era composta por oito enfermeiros, 31 técnicos de enfermagem, 26 auxiliares e um atendente (em processo de mudança para a categoria de auxiliar). O processo de trabalho em enfermagem, nesta unidade, integra trabalhadores de diferentes níveis técnicos e também de escolaridade. De acordo com Kurgant (2005), a enfermagem é uma classe que se caracteriza por uma divisão técnica e social no trabalho, definida por diferentes categorias de trabalhadores com diferentes níveis de formação, que utilizam um saber específico e instrumentos próprios como meio de realização do trabalho.

As equipes se dividiam em três turnos de trabalho, sendo manhã $(32,4 \%)$, tarde $(32,4 \%)$ e noite. Dos respondentes, cinco referiram cumprir 
duplo vínculo empregatício na mesma instituição, o que permitia que trabalhassem em jornada dupla e, portanto recebessem duplo salário. No período noturno, $35,2 \%$ se revezavam em duas equipes, trabalhando 12 por 36 horas de descanso.

Em relação ao vínculo empregatício com a instituição, os resultados apresentaram valor equivalente a 19,7\% com contratado temporário válido por um ano, e 80,3\% como efetivos.

A situação funcional das equipes era a seguinte: $94 \%$ exerciam suas atividades em situação plena; $6 \%$ eram readaptados, ou seja, encontravam-se impedidos de exercer plenamente as atividades profissionais por motivo de saúde, e, por isso, estavam realocados na própria unidade para desempenho de outras atividades.

Do total de questionários respondidos, 30,3\% possuem mais de um emprego, e todos são relacionados à área de saúde e 68,2\% trabalham somente no HURNP. Sabe-se que o trabalho em saúde é estressante, mas a dupla jornada é uma opção para complementar a remuneração salarial inadequada e insuficiente para sustentar a família. Isso os obriga a sobrecarregar-se de serviços, o que resulta em desgaste físico, podendo influenciar na qualidade de vida e, também, do exercício profissional (PARFARO; MARTINO, 2004).

A questão com possibilidade de múltiplas respostas a respeito do que é considerado qualidade na assistência de enfermagem, apresentou como retorno as seguintes referências: o domínio das técnicas (63\%), a ética no atendimento (51\%), atender ao paciente de forma plena $(57 \%)$, ter boa qualificação profissional (63\%), priorizar o paciente (43\%), humanização no atendimento (85\%), trabalhar sem sobrecarga no serviço $(45 \%)$ e ter disponibilidade de materiais e medicamentos para atender os pacientes (55\%).

Chenso et al. (2004) lembra que a crescente demanda por profissionais de enfermagem capacitados para prestar serviços de saúde hospitalar com qualidade, exige que os administradores dessas instituições não se limitem ao simples recrutamento e seleção de pessoas, mas invistam no desenvolvimento e orientação das potencialidades individuais e da equipe, na busca do atendimento com qualidade. A reciclagem contínua de conhecimentos, com suporte de informação dentro de um contexto multiprofissional, aprimora e desenvolve capacidades para o reconhecimento das necessidades humanas afetadas, por subsidiar a reflexão e as tomadas de decisões relativas à assistência de enfermagem.

Os dados mostram, também, que 31,8\% desconhecem o trabalho desenvolvido pelaACQAE; $53,0 \%$ conhecem parcialmente este trabalho e $15,2 \%$ assinalaram que conhecem. A existência de alta rotatividade de recursos humanos no setor, em razão de contratações temporárias, sugere um entrave aos profissionais para familiarizar-se com o processo de avaliação da ACQAE.

A questão central levantada no estudo foi a forma de repasse dos dados coletados pela ACQAE: $24,6 \%$ assinalaram como adequada; 44,6\%, insuficiente; $30,8 \%$, inadequada. Esses resultados reforçam a necessidade de mudança no processo de repasse das avaliações, visto que um nível melhor de satisfação apresentado pelas equipes poderia resultar em maior envolvimento e responsabilidade no acréscimo de qualidade ao cuidado prestado.

Questionados quanto à importância dos dados levantados pela ACQAE, consideraram importantes para a prática profissional $81,8 \%$; não importantes $9,1 \%$, e consideram indiferentes $9,1 \%$. Apesar de a maioria apontar os dados levantados pela ACQAE como relevantes, existia resistência por parte dos próprios profissionais em aderirem à educação continuada, como comentamos na introdução desse estudo. Empregar incentivos às potencialidades individuais e da equipe, assim como valorizar expressivamente um trabalho concreto de boa qualidade, pode ser uma estratégia usada pelo profissional responsável pelo repasse de resultados 
para reverter esse comportamento e estimular a conscientização da importância na qualidade da assistência.

Sobre a periodicidade em que a unidade deveria passar pela avaliação de qualidade, 35,4\% responderam mensalmente; $32,3 \%$ trimestralmente; $15,4 \%$ semestralmente e, anualmente, $16,9 \%$. Observa-se que há um percentual alto, acima de $30 \%$, que consideram que esta avaliação deve acorrer entre uma e duas vezes no ano, sugerindo a não valorização, ou desconhecimento do trabalho realizado pela ACQAE. Em relação a essa possibilidade, Meira e Oliveira (2004) salientam que, além de ter uma equipe bem preparada dentro da empresa, é necessário potencializar as capacidades humanas através de comunicação interna, para mantê-las sempre muito bem informadas, estimulando-os a executar um bom trabalho a partir do conhecimento sobre as metas da organização.

A figura 1 serve para demonstrar a preferência das equipes de enfermagem em relação aos profissionais tidos como capacitados para fazer o repasse de resultados da ACQAE.

A figura 1 corrobora a hipótese que motivou este estudo, mostrando que existe preferência por profissional preparado para repassar os resultados da ACQAE.

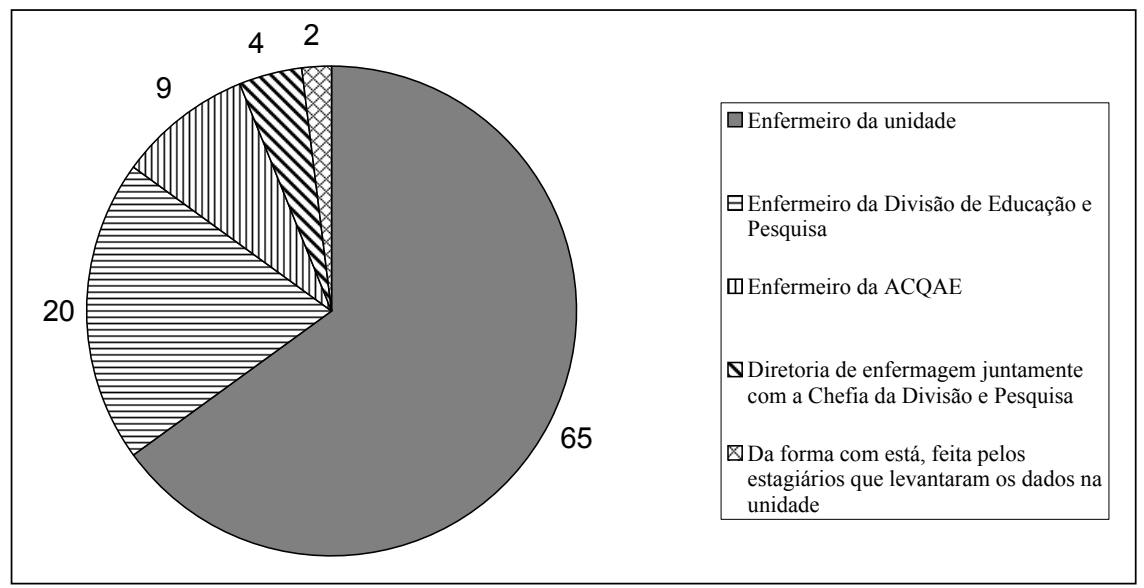

Figura 1. Porcentagem de pessoas consideradas adequadas para o repasse dos resultados dos relatórios de avaliação da unidade, Londrina, PR, 2007.

A satisfação de $2 \%$ dos componentes das equipes com a forma atual de repasse dos relatórios pela ACQAE sugere pouca credibilidade dos alunos na realização desse trabalho, por provável inexperiência prática. Mudanças no processo, visando ao maior envolvimento dos profissionais para melhorar o padrão de qualidade de assistência, poderiam otimizar esse resultado. Em relação aos $20 \%$ que apontam o enfermeiro da Divisão de Educação e Pesquisa como o profissional adequado para fazer o feedback, é suposto que mesmo a capacitação do profissional para exercer essa tarefa não se mostrou suficiente para satisfazer os anseios da maioria dos trabalhadores, que, provavelmente, elegeram o relacionamento inter-pessoal como um importante motivador para o processo de melhoria da qualidade de assistência. Isso se evidenciou na demonstração de $65 \%$ na preferência pelo Enfermeiro da unidade, revelando a importância desse profissional na efetivação do processo de repasse de resultados e confirmando a hipótese precursora deste estudo, que foi a necessidade de conhecimento teórico/prático do processo de enfermagem. Além de se apresentar como conhecedor da realidade do local de trabalho, a proximidade do enfermeiro com os componentes da equipe, permite uma maior percepção das qualidades e limitações individuais e do grupo, o que poderia alimentá-lo para desenvolver experiências de aprendizagem de interesse das pessoas envolvidas. Sentone (2005) salienta que a equipe de enfermagem 
deve estar comprometida, coesa e estimulada para propor estratégias criativas para a resolução de problemas relacionados à qualidade de assistência.

A melhoria de qualidade da assistência exige que os profissionais das equipes de enfermagem sejam motivados a buscar situações de ensinoaprendizagem, desenvolvendo a percepção de que são capazes de aprender sempre, desde que recebam orientações quando necessário. Nessa perspectiva, o enfermeiro da instituição deve planejar ações educativas, pois tem melhores condições de perceber a realidade e avaliar as necessidades dos integrantes da própria equipe. Ao realizar esses momentos de educação em serviços, poderá promover o aperfeiçoamento da equipe diante da evolução científica e tecnológica, elevando a competência e a valorização profissional e institucional (PASCHOAL, 2004).

Sobre o local mais adequado para o repasse dos resultados, $89,4 \%$ consideram que é a própria unidade, e 10,6\% fora do local de trabalho. $\mathrm{Na}$ escolha do melhor horário, 80,3\% consideraram que o repasse de resultados deve ser feito dentro do horário de trabalho e 19,7\% fora do horário de trabalho. Para 24\%, o melhor momento seria o horário de visita aos pacientes ou do lanche; para $9 \%$, nos plantões de final de semana; para $7 \%$ no meio da noite; e $60 \%$ não opinaram quanto ao momento ideal para o feedback. Os profissionais integrantes das equipes de enfermagem muitas vezes cumprem jornadas duplas de trabalho, e têm que sair às pressas para chegar ao outro emprego a tempo de cumprir a jornada programada. A possibilidade de residir longe do local de trabalho seria outro entrave para a participação das oficinas de educação em serviço, num horário ou momento diferente dos considerados acima. Percebe-se que houve um reforço para que sejam relevadas suas opiniões, quando os momentos adequados para o repasse estão aliados ao tempo de maior tranqüilidade dos profissionais no local de trabalho.

Em relação aos fatores que poderiam interferir na qualidade de assistência, todos assinalaram mais de uma questão, com $66 \%$ referindo os recursos humanos; 62\%, os recursos materiais; $54 \%$, os interesses pessoais; $43 \%$, a disponibilidade profissional; $60 \%$, o comprometimento profissional; $56 \%$, a estrutura física da unidade. A Resolução $n^{\circ}$ 293/2004 do Conselho Federal de Enfermagem COFEN (1998) que trata do dimensionamento do quantitativo mínimo dos profissionais de saúde nas instituições, visa a resolver a maioria problemas relativos aos recursos humanos, garantindo mais segurança e qualidade na assistência ao paciente, uma vez que a rotina dos serviços de enfermagem, caracterizada por uma diversidade de serviços repetitivos, com ritmo intenso, exige esforço físico e desgaste emocional, somado à sobrecarga de trabalho. Tudo isso, além de outros fatores que interferem na qualidade de vida dos profissionais, pode interferir no interesse, na disponibilidade e no comprometimento profissional. A distribuição quantitativa equilibrada de profissionais de enfermagem, na instituição, poderia estimular e fortalecer a qualidade na assistência.

Com relação aos recursos materiais, sabe-se que depende de previsão de uso, para posterior provisão, e que, em se tratando de hospital público, depende dos recursos governamentais nem sempre disponíveis. A estrutura física do HURNP faz parte de construções antigas, as quais sofreram inúmeras reformas ao longo de sua história. Com relação à unidade de internação masculina, o ajuste do ambiente físico visando proporcionar maior conforto físico e emocional aos pacientes e funcionários seria uma influência positiva em todo o processo de trabalho, estimulando a melhoria da qualidade de cuidados prestados.

\section{Considerações Finais}

O desenvolvimento da pesquisa demonstrou que o processo de feedback dos resultados das avaliações, precisa ser revisto no sentido de atender às expectativas da equipe, que levantou a necessidade de mudanças relativas à forma usada para atualização e educação continuada, além de 
demonstrar a falta de informação sobre a importância do trabalho exercido pela ACQAE.

A implementação de uma proposta inovadora, atendendo aos anseios dos envolvidos, pode facilitar o processo de compreensão e construção dos conhecimentos, facilitando o desenvolvimento da qualidade nos serviços. Desse modo, sugere-se a realização de estudo posterior à reestruturação deste processo, no sentido de verificar se ofeedback realizado pelo enfermeiro da unidade, como forma de educação continuada, resultará em melhora na qualidade da assistência dispensada pela equipe de enfermagem da unidade estudada.

\section{Agradecimentos}

Aos funcionários da Unidade Masculina do Hospital Universitário Regional Norte do Paraná, que permitiram a realização deste estudo.

\section{Referências}

ANTUNES, A. V.; TREVIZAN, M. A. Gerenciamento da qualidade: utilização no serviço de enfermagem. Revista Latino-Americana de Enfermagem, Ribeirão Preto, v. 8, n. 1, jan. 2000. Disponível em: <http://www.scielo.br/ pdf/rlae/v8n1/12432.pdf>. Acesso em: 15 mar. 2007.

BALBUENO, E. A.; NOZAWA, M. R. Levantamento dos tipos de repercussões resultantes da avaliação de desempenho em enfermagem hospitalar. Revista LatinoAmericana de Enfermagem, Ribeirão Preto, v. 12, n. 1, jan./fev. 2004. Disponível em: <http://www.scielo.br/ scielo.php?pid=S0104>. Acesso em: 10 ago. 2008.

BRASIL. Conselho Nacional de Saúde. Resolução n. 196, de1996. Diretrizes e Normas regulamentares de pesquisa envolvendo seres humanos. Brasília, DF, 1996.

BRAZ, M. G. Indicadores de qualidade na assistência domiciliar: uma proposta de indicadores de qualidade e desempenho. Rio de Janeiro: PRONEP, 2002. p. 1-14.

CHENSO, M. Z. B.; HADDAD, M. C. L.; SÊCCO, I. A. O.; DORIGÃO, A. M.; NISHIYAMA, M. N. Cálculo de pessoal de enfermagem em um Hospital Universitário do Paraná: uma proposta de adequação. Revista Semina: Ciências Biológicas e da Saúde, Londrina, v. 25, n. 1, jan./dez. 2004. Disponível em: <http://www.uel.br/ proppg/semina/index.php?arq=ARQ>. Acesso em: 10 ago. 2008.
CIANCIARULLO, T. I. $C \& Q$ : teoria e prática em auditoria de cuidados. São Paulo: Ícone, 1997.

CIANCIARULLO, T. I.; GUALDA, D. M. R.; MELLEIRO, M. M. (Orgs.). Indicadores de qualidade: uma abordagem perinatal. São Paulo: Ícone, 1998. (Série C \& Q).

CONSELHO FEDERAL DE ENFERMAGEM COFEN. Resolução n. ${ }^{\circ} 293$, de 2004. Estabelece normas para dimensionamento do quadro mínimo dos diferentes niveis profissionais de enfermagem para cobertura assistencial nas instituições de saúde, Rio de Janeiro, jan. 1998.

HADDAD, M. C. L. Qualidade da assistência de enfermagem: $\mathrm{O}$ processo de avaliação em hospital universitário público. 2004. Tese (Doutorado em enfermagem) - Escola de Enfermagem de Ribeirão Preto, Ribeirão Preto.

KURGANT, P. Gerenciamento em enfermagem. Rio de Janeiro: Guanabara Koogan, 2005.

MALIK, A. M. Pesquisa em, sobre e para os serviços de saúde no Brasil. Cadernos de Saúde Pública, Rio de Janeiro, v. 20, supll. 2, p. 161-63, 2004.

MEIRA, P.; OLIVEIRA, O. O endomarketing. 2004. Disponível em <http://endomarketing.com/artigo_ endomarketing.htm>. Acesso em: 25. out. 2007.

NEVES, J. F.; NEVES, M. C. P. Qualidade $e$ sustentabilidade. 2000. Disponível em <http://www. cnpab.embrapa.br/servicos/>. Acesso em: 20 mar. 2007.

NONINO, E. A. P. M. Avaliação da qualidade dos procedimentos de enfermagem - banho e curativo - segundo o grau de dependência assistencial dos pacientes internados em um hospital universitário. 2006. Tese (Doutorado) - Escola de Enfermagem de Ribeirão Preto, Universidade de São Paulo, Ribeirão Preto, 2006.

PARFARO, R. C.; MARTINO, M. M. F. Estudo do estresse do enfermeiro com dupla jornada de trabalho em um hospital de oncologia pediátrica de Campinas. Revista Escola de Enfermagem USP, São Paulo, v. 38, n. 2, p. 152-160, 2004.

PASCHOAL, A. S. O discurso do enfermeiro sobre educação permanente no grupo focal. 2004. Dissertação (Mestrado) - Universidade Federal do Paraná, Curitiba, 2004.

SENTONE, A. D. D. Análise dos indicadores da qualidade na elaboração da prescrição de enfermagem em uma unidade de internação de um hospital universitário público. 2005. Dissertação (Mestrado) Escola de Enfermagem de Ribeirão Preto, Universidade de São Paulo, Ribeirão Preto, 2005. 
Apêndice

Instrumento para coleta de dados

Data $\mathrm{N}^{\mathrm{o}}$

1. Categoria profissional:

1.1 ( ) Atendente de enfermagem

1.2 ( ) Auxiliar de enfermagem

1.3 ( ) Técnico de enfermagem

1.4 ( ) Enfermeiro

2. Idade:

3. Sexo:

3.1 ( ) masculino

3.2 ( ) feminino

4. Escolaridade:

4.1 ( ) Ensino fundamental incompleto

4.2 ( ) Ensino fundamental completo

4.3 ( ) Ensino médio incompleto

4.4 ( ) Ensino médio completo

4.5 ( ) Profissionalizante/ Técnico/Pós Médio incompleto

4.6 ( )Profissionalizante/ Técnico/Pós Médio completo

4.7 ( ) Superior incompleto

4.8 ( ) Superior completo

4.9 ( ) Pós-Graduação incompleta

4.10 ( ) Pós-Graduação completa

5. Estado civil:

5.1 ( ) casado/a

5.2 ( ) solteiro/a

5.3 ( ) separado/ divorciado

5.4 ( ) viúvo/a

5.5 ( ) outros

6. Número de filhos:

7. Tempo de atuação nesta instituição:

8. Turno de trabalho nesta instituição:

8.1 ( ) manhã

8.2 ( ) tarde

8.3 ( ) noite

9. Tipo de vínculo empregatício:

9.1 ( ) temporário

9.2 ( ) efetivo

10. Situação funcional:

10.1 ( ) situação funcional plena

10.2 ( ) readequado: impedido de exercer atividade temporariamente
( ) readaptada: impedido de exercer funções definitivamente

11. Possui mais de um emprego?

11.1 ( ) sim

11.2 ( ) não

Se sim, descreva-o:

12. Estuda no momento?

12.1 ( ) sim

12.2 ( ) não

Se sim, relate:

13. Escreva no espaço abaixo, o que você considera como qualidade na assistência de enfermagem:

14. Você conhece o trabalho desenvolvido pela Assessoria de Controle de Qualidade da Assistência (ACQAE) na sua unidade?

14.1 ( ) sim

14.2 ( ) não

14.3 ( ) em parte

15. Partindo do princípio de que a ACQAE tem como finalidade detectar os problemas que possam estar interferindo na qualidade de assistência de enfermagem, visando instrumentar os gerentes e o serviço de educação continuada na tentativa de solucioná-los, você considera que o método utilizado para o repasse dos resultados da avaliação da unidade para a equipe de enfermagem é:

15.1 ( ) adequada

15.2 ( ) insuficiente

15.3 ( ) inadequada

Comente sua resposta

16. Você considera os dados levantados pela ACQAE sobre a qualidade de assistência na sua unidade:

16.1 ( ) não são relevantes para o desenvolvimento da prática profissional

16.2 ( ) são importantes para o esenvolvimento da prática profissional

$16.3($ ) indiferente 
17. Na sua opinião, com que freqüência as unidades deveriam passar pelo processo de avaliação da qualidade de assistência de enfermagem desenvolvida pela ACQAE?

17.1 ( ) mensalmente

17.2 ( ) trimestralmente

17.3 ( ) semestralmente

17.4 ( ) anualmente

18. Quem seriam as pessoas mais indicadas para fazer esse repasse de resultados?

18.5 ( ) o enfermeiro da unidade

18.6 ( ) o enfermeiro da divisão de educação e pesquisa

18.7 ( ) a enfermeira da ACQAE

18.8 ( ) a diretora de enfermagem juntamente com a chefe da divisão e pesquisa

18.9 ( ) da forma como está, feita pelos estagiários que levantaram os dados na unidade

19. Qual local seria mais adequado para o repasse dos resultados obtidos na unidade para os membros da equipe de enfermagem?

19.1 ( ) na própria unidade

19.2 ( ) fora do local de trabalho

20. Qual o melhor horário?

20.1 ( ) dentro do horário de trabalho

20.2 ( ) fora do horário de trabalho

21. Em seu ponto de vista, que fatores poderiam interferir no resultado da qualidade de assistência de enfermagem?

2l.1 ( ) recursos humanos

21.2 ( ) recursos materiais

21.3 ( ) interesse profissional

21.4 ( ) disponibilidade profissional

21.5 ( ) comprometimento profissional

21.6 ( ) estrutura física da unidade
Anexo

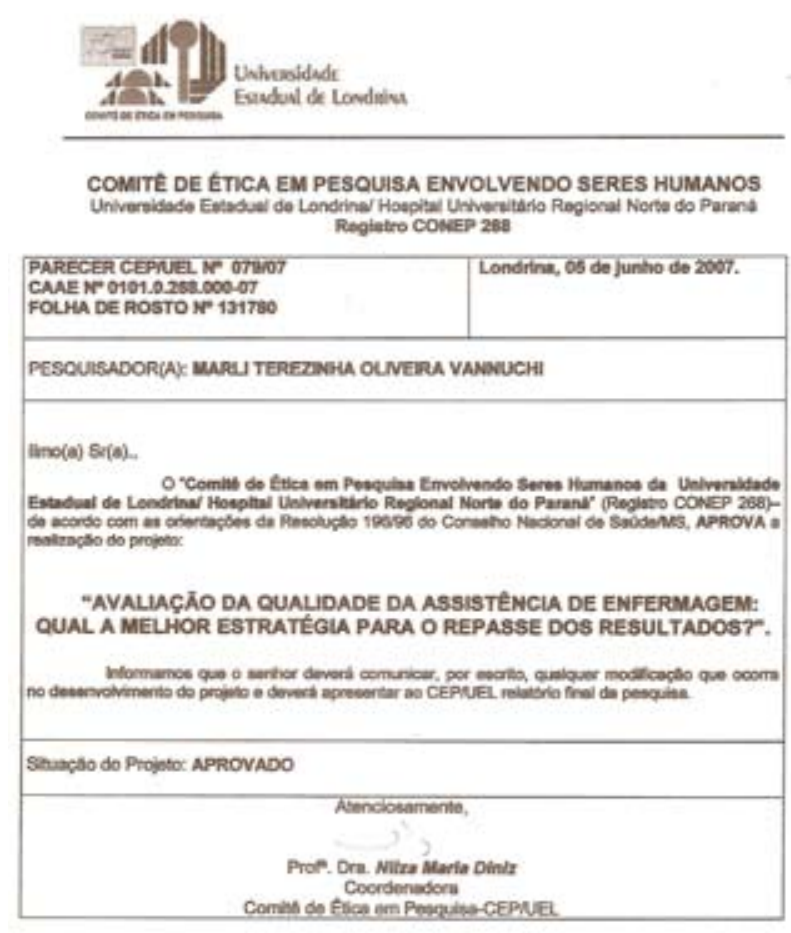

Cा1

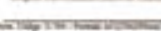

